\title{
EDITORIAL
}

\section{Uropathogenic bacteria leave a mark}

\author{
Wolfgang A Schulz
}

Urinary tract infections are common, obnoxious and in some cases even life endangering. They are most often caused by uropathogenic Escherichia coli. Persistent and seemingly recurrent infections may be caused by bacteria establishing intracellular reservoirs in the urothelial epithelium. A better understanding of the mechanisms involved in the complex interplay between host and pathogens is required for preventing and controlling these infections. A paper in this issue of Laboratory Investigation reports changes in gene expression in urothelial carcinoma cells following infection by uropathogenic bacteria which go along with the activation of DNA methyltransferase 1 and increased methylation at the CDKN2A gene. These pioneer findings should stimulate the further development of in vitro models for studying urothelial infections and prompt more extensive analyses of epigenetic alterations elicited by bacterial infections in the urinary tract.

Laboratory Investigation (2011) 91, 816-818; doi:10.1038/labinvest.2011.51

KEYWORDS: DNA methylation; epigenetics; urinary tract infection; uropathogenic $E$. coli; urothelium

\section{THE CHALLENGE OF URINARY TRACT INFECTIONS}

A pproximately 150 million cases of urinary tract infections occur worldwide annually. ${ }^{1}$ A prominent group among patients are young and otherwise healthy women. Appropriate treatment with antibiotics will cure many cases, but the disease will recur in about $25 \%$ of affected persons. Infections are often associated with inflammation and pain, and upon ascending to the kidney may cause pyelonephritis with renal damage and all its dire consequences.

Most commonly, urinary tract infections are caused by uropathogenic Escherichia coli. ${ }^{2,3}$ In order to establish acute or chronic infections, the bacteria have to express specific virulence factors. ${ }^{4}$ For instance, they require type I pili (fimbriae) for attaching to receptors on the urothelial surface strongly enough to withstand the shear force of the urinary flow. Research over the last decade has revealed that uropathogenic E. coli invade

Department of Urology, Heinrich Heine University, Düsseldorf, Germany Correspondence should be addressed to: wolfgang.schulz @uni-duesseldorf.de urothelial cells to replicate therein. Even worse, the bacteria may establish reservoirs that likely serve as prominent sources of seemingly recurrent infections. ${ }^{5}$

The interplay between host and pathogen during urinary tract infections is complex and modulated by genetic differences among human patients as well as uropathogenic bacteria. ${ }^{4,6}$ It is now established that both innate and adaptive immune responses contribute to clearance of the bacteria from the urothelial epithelium, but concomitant inflammation accounts for several disease symptoms. ${ }^{2,3,7}$ Recognition of pathogenic bacteria is achieved, among other mechanisms, by toll-like receptors such as TLR4. ${ }^{8,9}$ The pathogens in turn wield several mechanisms in order to evade or even exploit the defense responses provided the urothelial barrier and the immune system to establish and maintain infections. For instance, an important defense mechanism in the early infection phase is the exfoliation of upper layers of the infected urothelium, including the so-called umbrella cells with bacteria attached or internalized. ${ }^{2,3,7}$ This per se efficacious protective mechanism has the downside of exposing the underlying layers of the urothelium to more efficient infection.

Although many aspects of the interaction between uropathogenic E. coli and the cells of the urinary tract have been revealed, we are far from a comprehensive understanding of the pathogenic mechanisms involved. Elucidation of these mechanisms is obviously necessary to achieve progress in therapy and metaphylaxis, especially for ameliorating acute symptoms, avoiding 
progression towards renal disease and preventing recurrences.

\section{UROTHELIAL CARCINOMA CELL LINES AS INFECTION MODELS}

In the current issue of Laboratory Investigation Tolg et al ${ }^{10}$ report quite selective short-term changes in gene expression and epigenetic changes in urothelial carcinoma cells infected by uropathogenic E. coli. The paper is intriguing for several aspects. For one, the authors show that a relatively simple and straightforward experimental setup can be employed to unravel mechanisms involved in the interaction between pathogenic bacteria and urothelial cells. They used established urothelial carcinoma cell lines, which are easy to handle and retain features of urothelial differentiation. In fact, the many available cell lines from bladder cancers differ widely in the extent and pattern of urothelial differentiation and may also vary in the expression of pathogen recognition molecules such as TLRs. In particular, some of these cell lines-especially those derived from papillary urothelial tumors-have been reported to undergo stratification and a certain degree of terminal differentiation under certain culture conditions. A more faithful model would be reconstited normal urothelium in culture, which is feasible, if tedious. ${ }^{11,12}$ Therefore, varying and extending the present culture model should have great potential to provide additional insights into pathogenic mechanisms during urothelial infection.

Obviously, the experimental model system can similarly be exploited to analyze bacterial pathogenicity factors. In the present investigation, the authors compared fimbriae-positive E. coli with a strain lacking these attachment structures, which served as one general control throughout their study. Similar comparisons could be extended to other pathogenicity factors relevant to the 'in culture' situation.

\section{EPIGENETICS OF INFECTION AND CARCINOGENESIS IN UROTHELIAL CELLS}

Tolg et $a l^{10}$ made a novel intriguing observation using their experimental model. Successful infection led to quite specific changes in gene expression. In particular, however, the authors report an increase in DNA methyltransferase I (DNMT1) expression as well as activity, along with decreased expression of CDKN2A.

Accordingly, elevated DNA methylation could be detected in a CpG island of this important tumor suppressor gene. Expression of the DNA repair enzyme MGMT was likewise downregulated, but no change in methylation was detected at this gene. Of note, the change in CDKN2A methylation was relatively slight and it will be important to study a larger number of loci and additional incubation conditions to substantiate this finding. If so, it will be highly interesting to identify the bacterial factors eliciting increased DNMT1 expression and targeting to specific genes. Nevertheless, the observation provides- to my knowledge- the first piece of evidence that infection of urothelial cells by uropathogenic E. coli not only causes activation of innate immune signaling pathways and short-term changes in gene expression, but also may establish permanent epigenetic changes. Conceivably, these marks made by the bacteria might help to shape the cellular environment for persistent infections. If so, the speculation by Tolg et al that they might be used as a biological marker for susceptibility to recurrent infections may be correct.

Alterations of DNA methylation by pathogens are by no means unprecedented. ${ }^{13}$ For instance, there is very good evidence that Helicobacter pylori induces such changes in gastric cells. ${ }^{14}$ As during infection of urothelial cells by E. coli, the expression of MGMT is also compromised. ${ }^{15}$ Aberrant DNA methylation and other disturbances of epigenetic mechanisms initiated by pathogens is thought to contribute to the eventual development of cancer. From this point of view, the increased methylation at the tumor suppressor $C D K N 2 A$ is particularly intriguing. However, unlike in the case of $H$. pylori and the gastric epithelium, a causative relationship between bacterial infection and urothelial cancer development is not well supported by epidemiological data. ${ }^{16,17}$ Of note, infection by schistosoma parasites with its associated inflammatory cystitis is a well-known cause of bladder cancer, leading mainly to the otherwise rare squamous cell carcinoma subtype. ${ }^{18}$ The only published study on the topic-to my knowledgeindeed found a higher frequency of DNA methylation alterations in these cancers compared with conventional urothelial carcinomas. ${ }^{19}$ Moreover, it should be considered that the major mode of inactivation of CDKN2A in urothelial cancers is gene deletion, presumably elicited by chemical carcinogens. ${ }^{20}$ Still, the long-term consequences of epigenetic alterations induced by bacterial infections in the urinary tract ought to be evaluated for their potential importance in urothelial carcinogenesis. 


\section{DISCLOSURE/CONFLICT OF INTEREST}

The author declares no conflict of interest.

1. Foxman B. The epidemiology of urinary tract infection. Nat Rev Urol 2010;7:653-660.

2. Song J, Abraham SN. Innate and adaptive immune responses in the urinary tract. Eur J Clin Invest 2008;38(Suppl 2):21-28.

3. Weichhart $\mathrm{T}$, Haidinger $\mathrm{M}$, Hörl $\mathrm{WH}$, et al. Current concepts of molecular defence mechanisms operative during urinary tract infection. Eur J Clin Invest 2008;38(Suppl 2):29-38.

4. Wiles TJ, Kulesus RR, Mulvey MA. Origins and virulence mechanisms of uropathogenic Escherichia coli. Exp Mol Pathol 2008;85:11-19.

5. Dhakal BK, Kulesus RR, Mulvey MA. Mechanisms and consequences of bladder cell invasion by uropathogenic Escherichia coli. Eur J Clin Invest 2008;38(Suppl 2):2-11.

6. Svanborg C, Bergsten $\mathrm{G}$, Fischer $\mathrm{H}$, et al. Uropathogenic Escherichia coli as a model of host-parasite interaction. Curr Opin Microbiol 2006;9:33-39.

7. Hannan TJ, Mysorekar IU, Hung CS, et al. Early severe inflammatory responses to uropathogenic E. col predispose to chronic and recurrent urinary tract infection. PLoS Pathog 2010;6.

8. Ashkar AA, Mossman KL, Coombes $\mathrm{BK}$, et al. FimH adhesin of type 1 fimbriae is a potent inducer of innate antimicrobial responses which requires TLR4 and type 1 interferon signalling. PLoS Pathog 2008;4:e1000233.

9. Ragnarsdóttir B, Jönsson K, Urbano A, et al. Toll-like receptor 4 promoter polymorphisms: common TLR4 variants may protect against severe urinary tract infection. PloS One 2010:5:e10734.

10. Tolg C, Sabha N, Cortese R, et al. Uropathogenic E. coli infection provokes epigenetic downregulation of CDKN2A (p16INK4A) in uroepithelial cells. Lab Invest 2011:91:825-836 (this issue).
11. Tomlinson DC, Lamont FR, Shnyder SD, et al. Fibroblast growth factor receptor 1 promotes proliferation and survival via activation of the mitogen-activated protein kinase pathway in bladder cancer. Cancer Res 2009;69:4613-4620.

12. Varley $\mathrm{CL}$, Southgate J. Organotypic and $3 \mathrm{D}$ reconstructed cultures of the human bladder and urinary tract. Methods Mol Biol 2011;695:197-211.

13. Minárovits J. Microbe-induced epigenetic alterations in host cells: the coming era of patho-epigenetics of microbial infections. A review. Acta Microbiol Immunol Hung 2009:56:1-19.

14. Ding SZ, Goldberg JB, Hatakeyama M. Helicobacter pylori infection, oncogenic pathways and epigenetic mechanisms in gastric carcinogenesis. Future Oncol 2010;6:851-862.

15. Sepulveda AR, Yao Y, Yan W, et al. CpG methylation and reduced expression of O6-methylguanine DNA methyltransferase is associated with Helicobacter pylori infection. Gastroenterology 2010;138:1836-1844.

16. Murta-Nascimento $C$, Schmitz-Dräger BJ, Zeegers MP, et al. Epidemiology of urinary bladder cancer: from tumor development to patient's death. World J Urol 2007:25:285-295.

17. Wu X, Ros MM, Gu J, et al. Epidemiology and genetic susceptibility to bladder cancer. BJU Int 2008;102 1207-1215.

18. Abol-Enein $\mathrm{H}$. Infection: is it a cause of bladder cancer? Scand J Urol Nephrol Suppl 2008;218:79-84.

19. Gutiérrez $\mathrm{MI}$, Siraj $\mathrm{AK}$, Khaled $\mathrm{H}$, et al. $\mathrm{CpG}$ island methylation in Schistosoma- and non-Schistosomaassociated bladder cancer. Mod Pathol 2004;17: 1268-1274.

20. Florl AR, Franke $\mathrm{KH}$, Niederacher $\mathrm{D}$, et al. DNA methylation and the mechanisms of CDKN2A inactivation in transitional cell carcinoma of the urinary bladder. Lab Invest 2000;80:1513-1522. 